\title{
Innovaciones jurídicas, acceso, colaboración intercultural y pueblos indígenas en la educación superior
}

\author{
Legal innovations, access, intercultural collaboration and indigenous peoples in Higher Education
}

Víctor Ronald Zúniga Morales ${ }^{1}$

\section{Resumen}

El propósito de este ensayo es identificar innovaciones que se han venido dando en la Educación Superior Intercultural en Latinoamérica, para ello se analizaron una serie de referencias bibliográficas en el marco del primer encuentro de Doctorado en Estudios Interculturales dictado por la URACCAN. Encontramos que en las últimas décadas desde la conquista y la independencia de Latinoamérica los pueblos indígenas han mantenido sus luchas en cuanto a sus derechos y algunos de sus aportes innovadores se observan en cuanto a cambios en los marcos jurídicos nacionales e internacionales, creación de diversas instituciones de educación superior interculturales a través de diversas modalidades de colaboración, visiones, experiencias y propuestas novedosas. Estos son algunos de los resultados y con respecto a un nuevo sistema constitucional multicultural sobre nuevas formas del Estado con características únicas a escala mundial y una tendencia a ir forjando un Modelo de Universidad diferente, el actual ya no es pertinente al contexto latinoamericano, ya que se requieren otras maneras de hacer docencia, investigación y extensión, donde prevalezca la interculturalidad, colaboración y la innovación.

Palabras clave: Acceso; colaboración; educación superior; innovación; pueblos indígenas.

\begin{abstract}
The purpose of this essay is to identify innovations that have been taking place in Intercultural Higher Education in Latin America, for this a series of bibliographic references were analyzed within the framework of the first encounter of the Intercultural Studies Doctorate dictated by URACCAN. It is found that in the last decades since the conquest and independence of Latin America, indigenous peoples have maintained their struggles regarding their rights and some of their innovative contributions are observed in terms of changes in national and international legal frameworks, creation of various intercultural higher education institutions through various forms of collaboration, visions, experiences and innovative proposals. These are some of the results and with respect to a new multicultural constitutional system on new forms of the State with unique characteristics worldwide and a tendency to build a different University Model, the current one is no longer relevant to the Latin American context, since other forms of teaching, research and extension are required, where interculturality, collaboration and innovation prevail.
\end{abstract}

Keywords: Access; Collaboration; Higher Education; Innovation; Indigenous Peoples.

\footnotetext{
1 Máster en Desarrollo Rural con Enfoque Territorial. Director de Innovación y Emprendimiento de la Universidad de las Regiones Autónomas de la Costa Caribe Nicaragüense Email: victor.zuniga@uraccan.edu.ni, ORCID: https://orcid.org/0000-0002-4063-2219 


\section{Introducción}

Han pasado quinientos años de la colonización de Europa en el continente americano imponiendo dogmas y poderes de "viejo mundo" y a dos siglos de la independencia de Latinoamérica, esta última fue una gran oportunidad para las clases políticas hegemónicas, pero para los mestizos, mulatos, indígenas y negros, la nueva situación significo exclusión, imposición hegemónica y pobreza.

La situación anterior ha sido producto de modelos desarrollistas centrados en el crecimiento económico, la iglesia y los sistemas educativos han sido los principales instrumentos de sometimiento hegemónico, sin contar con la verdadera participación de los diversos pueblos presentes en Latinoamérica y sin reconocer que cada uno presenta una realidad y una forma de ser distinta.

Sin embargo, en las últimas tres décadas, la adopción del Convenio $169^{2}$ sobre pueblos indígenas y tribales en países de América Latina ha sido determinante para las luchas e iniciativas de los pueblos evidenciadas en innovaciones como el reconocimiento en las legislaciones, creación de diversas instituciones de educación superior intercultural de la región, modalidades sostenibles de colaboración intercultural, visiones y propuestas novedosas, experiencias y tendencias. Otros instrumentos claves en estos procesos se darán a conocer durante el transcurso de este artículo.

El objetivo de este artículo es identificar innovaciones que se han generado en la Educación Superior Intercultural de Latinoamérica y está organizado en tres secciones. La primera parte se presenta de forma muy resumida algunos cambios innovadores ${ }^{3}$ en las últimas décadas en Latinoamérica, centrados principalmente en instrumentos jurídicos (constituciones) y la existencia de una diversidad de experiencias de educación superior intercultural. En la segunda sección se comenta someramente la colaboración intercultural, algunas propuestas recientes de los pueblos indígenas y cual es su tendencia. Finalmente, en el último apartado se presenta a la Universidad de las Regiones Autónomas de la Costa Caribe Nicaragüense (URACCAN) que a sus 24 años es un modelo novedoso desde su creación, espíritu intercultural y algunos ejemplos de colaboración sostenida durante su vida institucional e innovaciones. Este ensayo incita a un cambio de modelo de las Universidades por estar en contextos multiculturales.

\section{Desarrollo}

\section{a. Panorama general sobre las constituciones, educación superior y acceso de pueblos indígenas en Latinoamérica}

La influencia europea en la formación de los Estados latinoamericanos generó por años la invisibilización de los pueblos indígenas en las legislaciones sin considerar que de acuerdo a datos de la CEPAL, en América Latina y el Caribe hay 826 pueblos indígenas que agrupan a más de 45 millones de personas, caracterizada por una enorme diversidad elementos que ha servido para importantes conquistas obtenidas a la fecha en la obtención de instrumentos jurídicos internacionales y nacionales de Latinoamérica como el Convenio 169 sobre pueblos indígenas y tribales (OIT, 1989) y la Declaración Universal de Derechos de los Pueblos Indígenas (NU, 2007).

Durante los últimos años estos instrumentos, han sido debatidos en distintos países lo que ha dado como resultado el reconocimiento del Multiculturalismo en las Constituciones Políticas Nacionales de países como El Salvador (1983), Guatemala (1985), Colombia (1991), Paraguay (1992), Perú (1993), Panamá

227 de junio de 1989 por la Organización Internacional del Trabajo (OIT).

3 Una innovación es la introducción de un nuevo, o significativamente mejorado, producto (bien o servicio), de un proceso, de un nuevo método u organización según Manual de OSLO. 
(1972 y 1994), Argentina (1994), Nicaragua (1987 y 1995), Guyana (1970 y 1996), Ecuador (1998), Honduras (1982 y 1999), Venezuela (1999), Costa Rica (1999), México (1992 y 2001), Brasil (1988 y 2002), Bolivia (1967 y 2004) y Chile (2009), estos cambios innovadores han dado como resultado el reconocimiento de la diversidad cultural y los derechos de los pueblos indígenas no así el de los pueblos afrodescendientes de América Latina, cuya invisibilidad todavía persiste (NU, 2006).

La creación de estos instrumentos innovadores a escala continental reconoce con mayor fuerza a los derechos de los pueblos indígenas y se asemejan por la alta presencia o participación de las organizaciones indígenas, aunque sea sumisa en algunos casos en las asambleas nacionales constituyentes de cada país. En esta época de cambio, en donde las constituciones políticas reconocen la diversidad étnica, cultural y lingüística, casi ningún país ha incorporado aspectos sustanciales en sus legislaciones sobre las demandas de reconocimiento, inclusión y participación de los pueblos indígenas y afrodescendientes sobre la Educación Superior.

A pesar de ello, existe una diversidad de experiencias de Educación Superior en/con/para pueblos indígenas impulsadas por varios actores entre ellos gobiernos, organismos no gubernamentales, fundaciones, universidades convencionales y mediante la creación de sus propias universidades comunitarias interculturales, esta diversidad de casos se ha implementado como producto de diversas modalidades de colaboración intercultural con los diversos actores antes mencionados.

A continuación, se presenta un panorama resumen de los diversos arreglos institucionales que se han dado dependiendo de cada contexto específico según lo plantea (Mato, 2018).

Programas para garantizar el acceso y graduación de estudiantes indígenas en universidades y otras IES "convencionales": Conocidos como programas de acciones afirmativas cuyo objetivo es la inclusión de personas indígenas y afrodescendientes, pero estas iniciativas no son suficientes, ejemplo de ellas son las becas de la Agencia Española de Cooperación Internacional para el Desarrollo (AECID),

- Programas especiales de grado, cursos certificados y centros en universidades y otras IES "convencionales": De estos programas hay una diversidad tanto a nivel nacional como internacional como los Programas de Educación Intercultural Bilingüe (EIB) en los Andes apoyado por la Cooperación Alemana ejecutado por la Universidad Indígena Intercultural.

- Proyectos de docencia, investigación y/o extensión o vinculación social desarrollados en universidades "convencionales" con la participación de pueblos indígenas. Esta es una de las prácticas que muy comúnmente se ve en las universidades convencionales, caracterizadas por acciones puntuales de ejecución de proyectos de docencia, investigación y extensión dependen de los fondos de cooperación y se ejecutan con participación de los pueblos.

Alianzas de universidades y otras IES convencionales con organizaciones de pueblos indígenas y/o con sus propias universidades de IES. Buscan desde un inicio la necesidad de negociar intereses y objetivos, casi siempre estas colaboraciones se dan por aspectos de accesibilidad o presencia geográfica.

- Universidades e IES interculturales e indígenas. Producto de la todavía influencia colonizadora que se aprecia en las universidades convencionales y el rezago de la interculturalidad, calidad, pertinencia y relevancia de la educación superior es que se han creación universidades interculturales por los propios pueblos. Caracterizados por integrar conocimientos, saberes, prácticas, sueños y 
aspiraciones de los pueblos, así como conocimiento "científico" atienden principalmente mas estudiantes indígenas que no indígenas, esto última contradictoria con la interculturalidad.

En general estamos frente a un contexto diferente novedoso y de gran oportunidad, lo peor es no consensuar como avanzar en el marco conceptual e implementación de la Educación Superior Intercultural como proyecto político de vida de la región.

\section{b. Interculturalidad, colaboración, propuestas y tendencias}

Al finalizar la segunda década del Siglo XXI, el mundo actual está en un proceso de cambios acelerados, complejos, cambiantes, de muchos retos y oportunidades. Estos retos cobran mayor significancia en Latinoamérica si se considera la diversidad de pueblos en la región que la hacen rica en culturas, saberes, tradiciones, prácticas, valores étnicos y maneras diferentes de ver, interpretar y vivir del mundo (Hooker, 2018).

En las últimas décadas se han venido dando la aparición de los pueblos demandando sus agendas de derechos generando nuevos modos de relación institucional con diversos actores, una de las demandas es la descolonización de la educación, los avances se ven expresados en distintos instrumentos jurídicos internacionales expresados principalmente en el derecho a una educación realista, respeto a la diversidad e interculturalidad.

En este contexto en Centroamérica se observan una diversidad de experiencias universitarias creadas desde distintas modalidades sostenibles de colaboración intercultural ya expresadas en el apartado anterior, respondiendo a tendencias generales (Mato, 2008 y 2012), esta es una región con marcadas características sociales, ambientales, políticas, económicas y culturales, representando el $7 \%$ en biodiversidad, 60 pueblos originarios y afrodescendientes en casi el $50 \%$ del territorio y economías entre el 4 a $5 \%$ pero con altos grados de desigualdad, siendo los pueblos indígenas y afrodescendientes los colectivos mas excluidos, marginados y poco disfrute de sus derechos básicos por el incumplimiento permanente de los gobiernos con compromisos internacionales y la perpetuidad de visiones coloniales que impiden posibilidades de diálogos de saberes.

La región centroamericana cuenta con más de 200 universidades de las cuales solo 22 (10\%) son públicas y unas 5 (2\%) se definen comunitarias, indígenas interculturales (Zúñiga, 2018). Este comportamiento de proliferación de universidades, así como el incremento de matrícula estudiantil es un comportamiento generalizado para toda Latinoamérica el reto sigue siendo que tanto programas y universidades sean contextualizadas con pertinencia cultural

En este contexto la educación superior está en procesos de transformación universitaria. Así lo vislumbró la "Declaración Mundial sobre la Educación Superior para el siglo XXI", aprobada en París en 1998 cuando expresaban que las universidades debían emprender las reformas más radicales que jamás antes hayan enfrentado, porque todavía persisten desigualdades, diferencias discriminación, inequidades y el reconocimiento de las diversidades. Ante estos planteamientos las universidades deben jugar un papel fundamental, ya que son llamadas a desempeñar ese roll desde la interculturalidad. El problema de hoy en día es que hablar de interculturalidad se hace desde distintas visiones, enfoques que responden a contextos diferentes, desde experiencias e intereses diferentes por tanto es bueno reflexionar sobre ¿Qué entendemos por interculturalidad?

Hablar de interculturalidad, muchas veces se hace desde el discurso, sin realmente profundizar en los grandes desafíos que representa y es usada desde una diversidad de significados, pero ¿Desde dónde viene esta polisemia de la interculturalidad? Esta polisemia tiene su origen desde las mismas fuentes en 
sus distintos contextos creando a nivel global confusión, significados diferentes como es el caso desde las sociedades anglosajonas en comparación con Latinoamérica.

Dietz (2017), plantea que en "algunas sociedades, la interculturalidad se utiliza para referir a la diversidad provocada por la migración, mientras que en otras la misma noción se aplica para las interacciones entre pueblos indígenas y descendientes de colonizadores" (p.192), la interculturalidad proviene de diversas fuentes, en Latinoamérica vemos que hemos tenido mucha influencia de la vertiente anglosajona centrada en el multiculturalismo que gestiona la diversidad para que esta deje de ser un problema, asignando cuotas de participación a los pueblos, al final estas políticas tratan a las minorías para que sigan siendo minorías.

Esto exige a la educación superior latinoamericana y centroamericana a consensuar un entendimiento de la interculturalidad para fortalecer la diversidad cultural como una oportunidad más que como obstáculo o factor de división, donde las sociedades del conocimiento son parte de nuestro modelo educativo.

Esto se fortalece con lo planteado por Mato (2009), expresado como “... un cierto tipo de mirada (a las relaciones) que puede aplicarse tanto al análisis de relaciones de colaboración, como de conflicto y que cualquier acción debe partir de reconocer que hay diversidad de contextos y por tanto de prácticas intelectuales y de saberes" (p.44), a lo cual complementaría que se aseguren condiciones mínimas en igualdad de condiciones para dialogar.

\section{Colaboración Intercultural “Es necesario empezar todo o casi todo”}

La innovación en los procesos de docencia, investigación y extensión, funciones sustantivas de las universidades- es una necesidad urgente y en la que los saberes ancestrales de los pueblos podrían contribuir -como principal fuente a la creación de nuevos conocimientos.

Históricamente Latinoamérica ha estado sometida bajo dolorosas y fuertes consecuencias por diversas visiones del mundo, lo que nos ha llevado a cuestionar en las universidades ¿Qué tipos de conocimiento producir, para qué y cómo? Las instituciones educativas insisten en enseñar contenidos descontextualizados, irrelevantes, poco utilizables, sin interrelación con la naturaleza y el cosmos. Sin embargo, lo que el mundo moderno necesita desesperadamente es una educación más pragmática, cuyos contenidos puedan utilizarse y aplicarse en la solución de los problemas que enfrentan los estudiantes, profesores y el liderazgo comunitario.

Si las universidades son la cuna o casa mayor del conocimiento donde se cultivan a los nuevos talentos que asumirán el bienestar de las sociedades, es necesario que aparte del entorno científico-académico, también conozcan sobre otras corrientes de saberes ancestrales a lo cual somos herederos.

La ciencia es más que producción, es un diálogo social-cultural-natural, porque todo está en la naturaleza, hemos caído en un estado de no cuestionar el paradigma tradicional porque nos enseñaron a implementar recetas -reglas- para poder acceder al conocimiento científico; sin embargo, hay otras maneras de hacer ciencia donde no prevalece un solo saber, donde no sólo los científicos son los grupos del saber. El problema es que cuanto los científicos nos volvemos arrogantes y poco permeables con otros sistemas de creencias, las sociedades toman otras alternativas irracionales muchas veces.

Para ello la educación superior intercultural propone desarrollar formas de colaboración intercultural que parten del reconocimiento y valoración de las diferencias para la producción de conocimientos no exenta de problemas, conflictos y desafíos. La colaboración intercultural permite tomar en cuenta el aprendizaje situado en y por la práctica, importante para la transmisión y reproducción de conocimiento tradicionales, 
su recreación y generación de innovaciones, esto también es crucial para la apropiación de conocimiento y destrezas propias de la llamada cultura occidental-moderna.

La Colaboración intercultural quiere decir establecer y sostener diálogos y relaciones interculturales de valoración y colaboración mutuas, que sean de doble vía. Diálogos y formas de colaboración honestas y respetuosas, de interés recíproco, que partan de reconocer que hay diversidad de contextos y de prácticas intelectuales y de saberes,(Mato, 2008). Producto del ejercicio de la colaboración intercultural se presentan en forma resumida propuestas innovadoras colectivas de relaciones interinstitucionales de universidades, otras instituciones de educación superior, gobiernos, redes y aportes de movimientos sociales e indígenas como son las declaraciones de las Conferencia Regional de Educación Superior.

La primera declaración (CRES, 1996), orienta que la educación superior debía preservar y fortalecer la identidad cultural de la región, a partir de acá las IES aumentan acciones afirmativas a través de asignación de cupos cuidando que el sistema ancestral no fuera a lesionar el sistema educativo convencional. La segunda declaración (CRES, 2008), mandata que el reto no es incluir estudiantes indígenas y afrodescendientes en la IES, es transformar estas universidades que sean pertinentes con la diversidad cultural, inclusión de cosmovisiones, conocimientos, saberes, practicas, sueños y aspiraciones.

En la reciente declaración (CRES, 2018), se ha permeado los términos de interculturalidad y se ve mejor comprendido lo cual se refleja no sólo como un término sino como un eje temático, esto como producto de un trabajo de vida teniendo como grandes protagonistas a una serie de actores indígenas, afrodescendientes, mestizos que ha venido implementando colaboraciones interculturales efectivas para obtener los avances logrados a la fecha.

En general existe una tendencia a diseñar un Modelo de Universidad diferente, el actual ya no es pertinente con el contexto de Latinoamérica, se requieren otras maneras de hacer docencia, investigación, extensión donde prevalezca la interculturalidad, colaboración intercultural y la innovación.

\section{c. La experiencia innovadora de la URACCAN}

La universidad de las Regiones Autónomas de la Costa Caribe Nicaragüense (URACCAN), es una universidad novedosa, se autodefine como "comunitaria intercultural", fue creada por un grupo de hombres y mujeres líderes indígenas, afrodescendientes y mestizos costeños de las Regiones Autónomas de Nicaragua

para ejercer el ejercicio pleno del derecho de los pueblos a una educación superior. Su carácter comunitario está determinado en el acompañamiento de los procesos de autogestión comunitaria para la profundización de la autonomía en la diversidad.

En la URACCAN la interculturalidad es concebida como un proceso permanente de construcción, establecimiento y fortalecimiento de espacios de diálogo, comunicación e interacción horizontal de doble vía, entre personas, comunidades y pueblos de diferentes culturas, también comprendida como el desarrollo de procesos de toma de decisiones conjuntas en igualdad de condiciones, siendo su finalidad la promoción y práctica de la equidad, el respeto, la comprensión, la aceptación mutua y la creación de sinergias para el establecimiento de sociedades inclusivas, no discriminatorias y libres de racismo, (URACCAN, 2015).

La URACCAN es una larga aspiración de los pueblos indígenas, afrodescendientes y mestizos costeños hecha realidad como producto de distintos procesos de colaboración intercultural y arreglos institucionales que se identifican a lo largo de su historia coincidiendo con lo planteado en la primera parte de este artículo. 
- Programas para garantizar el acceso y graduación de estudiantes indígenas en universidades y otras IES "convencionales": Antes de las universidades comunitarias interculturales de la Costa Caribe y todavía en la actualidad se mantiene cupos para el acceso de estudiantes indígenas en las universidades convencionales situadas en la capital del país.

- Programas especiales de grado, cursos certificados y centros en universidades y otras IES "conven-

cionales": Durante los 70 un grupo de hombres y mujeres costeños se unieron con la Universidad Nacional Autónoma de Nicaragua queriendo establecer una sede universitaria a través de una oferta temporal y por diversos motivos esta iniciativa no funcionó con normalidad afectando a los estudiantes que ingresaron.

- Proyectos de docencia, investigación y/o extensión o vinculación social desarrollados en universidades "convencionales" con la participación de pueblos indígenas: En los 80 esta iniciativa escala nuevamente a un Programa Universitario de Educación a Distancia (PRUEDIS), nuevamente no se tuvieron los resultados deseados por diversos motivos, quedando pendiente la culminación de estudios por alumnos y alumnas.

- Universidades e IES interculturales e indígenas: Con el apoyo de la cooperación internacional y recursos propios de los fundadores, URACCAN inicia operaciones en 1995 con los 120 estudiantes de ambas Regiones Autónomas procedentes del PRUEDIS.

- Alianzas de universidades y otras IES convencionales con organizaciones de pueblos indígenas y/o con sus propias universidades de IES. Desde su creación la URACCAN se ha visto inmersa en una serie de colaboraciones con diversos actores teniendo experiencias insatisfactorias como muy satisfactorias, tal es el caso con la cooperación de SAIH-Noruega con quienes todavía se colabora. A sus 24 años cada actor ha buscado desde un inicio la necesidad de negociar intereses, objetivos particulares y colectivos.

Algunas innovaciones realizadas con y para los pueblos indígenas, afrodescendientes y comunidades étnicas están en el Modelo de Salud Autonómico Intercultural, Sub-sistema Educativo Autonómico Regional, Plan de Forestería Comunitaria para la Costa Caribe, propuesta de indicadores de autoevaluación para universidades comunitarias intercultural en el marco de la RUIICAY, perspectiva intercultural de género como enfoque, el mismo Modelo de Universidad Comunitaria Intercultural en un sistema convencional, actualmente se cuenta con mas de 1000 graduados, muchos de ellos ocupando cargos de dirección e intermedios en el gobierno, ONG, empresas privadas, organizaciones de base comunitarias, sociedad civil y en las mismas universidades

\section{Conclusiones}

Para finalizar vemos que hoy en día el nuevo contexto multicultural reconocido de Latinoamérica es producto de las luchas de movimientos sociales e indígenas. Este nuevo sistema constitucional multicultural resulta ser novedoso sobre nuevas formas del estado con características únicas a escala mundial, la presencia de una diversidad de experiencias institucionales de educación superior intercultural no solo contribuyen al acceso de pueblos indígenas, también reflejan propuestas innovadoras en cuanto a calidad, pertinencia, relevancia y sedan bajo distintas modalidades novedosas de colaboración intercultural y en el caso de URACCAN esta es una universidad Comunitaria Intercultural en un Sistema Convencional al ser parte del Consejo Nacional de Universidades (CNU) lo que complejiza los procesos de gestión de la educación superior intercultural. A futuro se plantea profundizar en el modelo de innovación y emprendimiento con perspectiva intercultural de género en la primera universidad comunitaria intercultural. 


\section{Lista de referencia}

CEPAL. (2014). Los pueblos indígenas en América Latina. Avances en el último decenio y retos pendientes para la garantía de sus derechos. 1a ed., Santiago de Chile, ONU.

CRES. (1996). I Conferencia Regional sobre Políticas y Estrategias para la Transformación de la Educación Superior en América Latina y el Caribe. Centro Regional para la Educación Superior de la República de Cuba. (CRESALD, ahora IESAL).

CRES. (2008). Declaración y Plan de acción de la Conferencia Regional de Educación Superior en América Latina y el Caribe. Organización de las Naciones Unidas para la Educación la Ciencia y la Cultura. Instituto Internacional de la UNESCO para la Educación Superior en América Latina y el Caribe.

CRES. (2018). Declaración de la III Conferencia Regional de Educación Superior para América Latina y el Caribe. Organización de las Naciones Unidas para la Educación la Ciencia y la Cultura. Instituto Internacional de la UNESCO para la Educación Superior en América Latina y el Caribe.

Dietz, G. (2017). Interculturalidad: una aproximación antropológica. Revista Perfiles Educativos, 39(156), 192-207. Editorial Alejandro Márquez Jiménez. México.

Hooker-Blandford, A. (2018). Universidades e instituciones de educación superior indígenas, interculturales, afrodescendientes y comunitarias en América Latina. En: Daniel Mato (coord.). Educación superior, diversidad cultural e intercultural en América Latina. Págs.: 299 - 321. Editoriales: UNESCO-IESALC y UNC. Impreso en Argentina por la Universidad Nacional de Córdoba (UNC).

Mato, D. (2008). No hay saber "universal", la colaboración intercultural es imprescindible. ALTERIDDES, 2008, 18 (35). (Pág.: $101-116)$.

Mato, D. (2009). Contextos, conceptualizaciones y usos de la idea de interculturalidad. En: Miguel Aguilar, Eduardo Nivón, María Portal, Rosalía Winocur, coord...Pensar lo contemporáneo: De la cultura situada a la convergencia tecnológica. (Págs.:28 - 50). Anthropos Editorial. Barcelona, España.

Mato, D. (2018). Educación Superior y Pueblos Indígenas: Experiencias, estudios y debates en América Latina y otras regiones del mundo. Revista del Cisen Tramas/ Maepova, 6 (2), (Págs.: 41 - 65).

Mato, Daniel (Coord) (2012). Educación Superior y Pueblos Indígenas y Afrodescendientes en América Latina. Normas, Políticas y Prácticas. Caracas, Venezuela: UNESCO-IESALC.

Mato, Daniel (Coord). (2008). Diversidad cultural e interculturalidad en educación superior. Experiencias en América Latina. Caracas, Venezuela: UNESCO-IESALC.

OIT. (1989). Convenio 169 sobre pueblos indígenas y tribales.

UNESCO, IESALC. (2006). Informe sobre la Educación Superior en América Latina y el Caribe. 2000 - 2005. La metamorfosis de la educación superior. Primera edición. Caracas.

UNESCO. (1998). Declaración Mundial sobre la Educación Superior en el siglo XXI: Visión y Acción. Conferencia Mundial sobre la Educación Superior. Paris, Francia, 9 de octubre de 1988. 
URACCAN (2016). Perfil institucional. URACCAN: Universidad Comunitaria Intercultural. Universidad de las Regiones Autónomas de la Costa Caribe Nicaragüense. Managua.

Zúñiga, X. (2018). Persistencias coloniales, aperturas y desafíos para la educación superior en Centroamérica. En: Daniel Mato, coor. Educación Superior, Diversidad Cultural e Interculturalidad. Págs.:117 - 142. Editoriales: UNESCO-IESALC y UNC. Impreso en Argentina por la Universidad Nacional de Córdoba (UNC). 